not satisfy all the delegates. Several relatives of mentally ill people expressed their frustration at the inability of hospital staff to accept responsibility for providing for the needs of a sick family member. These carers wanted more help now, not just a promise of improvements in the future.

Judy Weleminsky, Director of the NSF, pointed to the lack of detail in the government's proposals. Looking back to the formation of the NSF in 1971, families of the mentally ill then were desperate for more support and information. Services available for care of psychiatric patients have since deteriorated: the number of hospital beds has halved, yet less than one in ten has been replaced by equivalent community care facilities. Family members confronting 'community neglect' feel anguish and neglect. Mrs Weleminsky conceded that although there was much wrong with the old style mental hospitals, such institutions did offer a home, support and treatment for patients. Today if hospital admission occurs it is often too late and too short.

Mrs Weleminsky expressed some particular concerns over the White Paper, questioning the government's commitment to the mentally ill and whether it would show action to meet its words. Previously Roy Griffiths emphasised the importance of having clear direct responsibility within the Health Service; abdication of such responsibility fails the mentally ill. Mrs Weleminsky would like to see a national plan and a Minister for Community Care to avoid the growth of haphazard and patchy services. She particularly encouraged health planners to listen more to sufferers and carers and to try and engage them in their discussions. More specifically, she discussed the issue of resources. Based on Professor Julian Leff's research (TAPS, 1990) and using a lower estimate of say $£ 10,000$ per year to provide a minimum level of support out of hospital for one person, by 1991 the government could be spending at least $£ 250$ million on care of the mentally ill in the community. Dr James Birley, President of the College, emphasised the scale of planning necessary. Directors of health and social services would have to get together and make joint plans on how best to meet the needs of their local populations so that individual patients get optimum care. For any one patient the health and social care required are indivisible and it is our responsibility to develop facilities to meet those needs. Such moves demand extensive research for, "How can anyone devise community care plans for the future when we do not know how many people will need that care and what kind of care that should be?" (Wallace, 1989).

\section{References}

Department of Health (1989) Caring for People: Community Care in the Next Decade and Beyond. Cmd 849. London: HMSO.

Proceedings from the Fourth Annual Conference of the Team for the Assessment of Psychiatric Services (TAPS), held at St Bartholomew's Hospital Medical College, July 1989. London: NETRHA (in press).

WALlace, M. (1989) Mental Health Organisations express concern at White Paper on Community Care. News release, 16 November 1989.

\title{
Computers in Psychiatry Special Interest Group - 2nd Annual General Meeting
}

\author{
StePhen Wood, Consultant Psychiatrist and Senior Lecturer; and Michael Sinclair, \\ Research Psychologist, Mental Health Advice Centre, 1 Southbrook Road, \\ London SE12 8LH
}

If three psychiatrists are needed to change a light bulb - one to change the bulb and two to interpret the experience - how many does it need to operate a computer? Judging by the attendance at the 1989 CIPG meeting, held at Keele University in October, the answer is about 54 .

Psychiatrists have been slow off the mark to explore and exploit computer applications, partly because of the nature of their craft which does not readily lend itself to the limitations imposed by simple algorithms, but also because psychiatrists by their nature and training are probably the least technologically minded members of the medical profession. However there are exceptions to every rule, and many of them gathered at Keele to share their knowledge and exchange ideas. The participants 
hailed from as far afield as Bermuda, Portugal, Limerick and the Isle of Lewis but surprisingly included relatively few from London in general and De Crespigny Park in particular. Technology it seems takes time to travel.

The meeting comprised lectures, workshops and practical demonstrations which were presided over by their inventors and proprietors rather like jars of home made jam at a WRVS jumble sale. The ethos too was reminiscent of the voluntary tradition, with many software packages being offered free or at a nominal charge, and only the most acquisitive adopting the values of the Enterprise Culture and seeking a commercial return on the many hours of programming that goes into the development of even a simple application, let alone a case register or questionnaire generator. Compared to only two or three years ago MSDOS and fast and expensive IBM clone personal computers were much in evidence, the few surviving BBC machines attracted the sort of nostalgia usually reserved for traction engines or vintage Morris Minors.

Nostalgia was also in evidence in the key note address given by the group's president Roger Bloor, a pioneer of psychiatric computing, who brought along his first home made computer, a Heath Robinson affair complete with valves, cobwebs and $1 \mathrm{~K}$ of random access memory. From this unpromising start Bloor described his growing infatuation with computers, culminating in a case register which he designed for the RAF. This proved to be so useful that it was hijacked by his superiors and expanded to the point where it became so ravenous for data input, and so parsimonious in its output, that its usefulness evaporated. Bloor warned against being seduced by creeping technological elegance at the expense of the chastity of clinical utility.

The Director of the King's Fund Audit Project, Charles Shaw, gave a glimpse of the brave new world which we will all inhabit after the White Paper, where the absence of auditing procedures and systems will be assumed to imply poor practice, if not incompetence. Pramod Jauhar from Glasgow described a depot neuroleptic and lithium monitoring system in use for several years, an impressive portent of things to come in the era of Working for Patients. Other case registers and case management systems which monitor the care of the seriously mentally ill in the community were demonstrated by David Martindale from Hackney, Stephen Wood and Mike Sinclair from Lewisham and Jason Taylor from Hampstead.

Case registers aside, one of the most interesting applications was described by Hutch Curry of the Burden Neurological Institute in Bristol, which uses BBC micro-computers loaned to the patient and linked by modem to the Institute to deliver visuospatial and lexical tasks to patients with static brain damage. It is in the area of graphics and colour, upon which effective educational and rehabilitative programmes depend, that the BBC still holds sway.

As an example of how sophisticated do it yourself software can be, Gyles Glover of the Westminster Hospital described an advanced questionnaire driver which can not only administer the PSE and GMS but also run CATEGO and AGECAT, and present the results in graphical format. The whole system can be run on a lap-top PC and should save many hours of research time, into the bargain saving several rainforests and helping to reduce the greenhouse effect.

At the first annual meeting of the CIPG in 1988 Professor Jim Hedlund started his address with a quotation from Griest: "We do not need another generation of computers or even of programming languages. We need a generation of clinicians who will take the tools presently available and apply then with care, ingenuity, diligence, and patience to difficult mental health problems." The Group's second meeting showed that this process is beginning to occur. Those wishing to join in should contact the secretary, Martin Briscoe, at Wonham House Hospital, Exeter. 